\title{
ORIENTATIONAL ORDERING FOR SYSTEM OF ELLIPSOIDAL MOLECULES DISPERSED IN CUBIC LATTICE
}

\author{
R. Mejdani* \\ International Centre for Theoretical Physics, Trieste, Italy \\ (Received January 4, 1996; revised version August 28, 1996)
}

\begin{abstract}
We represent here some results for a model of migrating and nonmigrating ellipsoidal molecules dispersed in a cubic lattice, describing: the orientational ordering in nematics, the possibility of a biaxial ordered liquid state, the orientational ordering in solid hydrogen (ortho- $\mathrm{H}_{2}$ or para- $\mathrm{D}_{2}$ ) or the orientational glassy structure (the "higher rank"glass state). Through this kind of molecular ensemble (in the case of the system of ellipsoidal and spherical molecules) it is also possible to study the orientational and positional orderings in binary mixtures and to obtain the so-called plastic state.

PACS numbers: $05.50 .+\mathrm{q}, 64.60 . \mathrm{Cn}, 64.60 . \mathrm{My}, 64.70 . \mathrm{Md}$
\end{abstract}

\section{Introduction}

A system consisting of anisotropic molecules exhibits two kinds of long-range ordering: long-range ordering of the centres of mass of the molecules or positional ordering and long-range ordering of the orientations of the molecules or orientational ordering [1-6]. When this system "melts" into an isotropic liquid usually both kinds of ordering disappear at the same temperature. Alternatively, it is possible that one of two ordering types survives until a transition temperature is reached. In case when it is the long-range orientational ordering which survives, we have a liquid crystal, e.g., a nematic or another anisotropic system (without positional ordering) as the solid hydrogen [7]. Another example is plastic crystal or plastic state, which differs from liquid crystals in that the first ordering mode which reaches zero is the orientational one, i.e., the centres of mass of the molecules still form a regular crystal lattice or sublattice, while their orientations display some degree of disorder $[8,9]$.

The ordering phenomena in liquid crystals have their features owing to the variety of the symmetries of the different phases. These features complicate theoretical considerations and numerical simulations, imposing restrictions on methods

\footnotetext{
*Permanent address: Department of Physics, University of Tirana, Albania.
} 
which are of proved validity in other cases. However, during these two last decades, instead of the computer calculations of the "mean-field" type (which neglect angular correlations between neighbouring molecules, certainly important at the actual physical densities and particularly near the transition point), a lot of work is done using models where the shape of molecules is represented by a hard body and the system of hard bodies is seen as one of the simplest idealizations of a molecular system [10-12]. Therefore, the simplest way to include the short-range molecular correlations (translational - crucial in fluid phases, and orientational - important in plastic crystals), coming from the repulsions of molecular cores, is offered by these hard-core systems. A review of results obtained for hard convex molecules is presented in [11]. Some results concerning a two-dimensional system of non-convex molecules (hard cyclic pentamers) are given in [13], where the singlet distribution function studied in detail, reveals a considerable coupling between translational and rotational molecular motions.

In spite of very simple interactions, e.g., infinite when any two bodies (molecules) overlap and zero otherwise, hard body systems are analytically intractable and most of the nontrivial results concerning their thermodynamic properties come from computer simulations. In particular, the simulations demonstrated that hard body systems exhibit melting transition and may form liquid crystalline phases when the body is highly non-spherical or plastic phases when the body is globular. By a proper choice of the shape of the body, one can model many of the existing thermodynamic phases in nature (as, e.g., monodisperse polystyrene latex by rigid spheres, tobacco mosaic virus by rigid rods, thin plate of kaolinite by rigid platelets, DNA, polymers by semiflexible chains, amphiphilic bilayer by semiflexible surfaces, etc.) (see Refs. [14-16] and references therein). Following the original work performed using models with purely steric interactions [10-12], there has been a growing interest in computer simulations of liquid-crystalline systems using models with "soft" potentials [12], where the various single-site anisotropic forms available continue to offer a productive route in studying order in liquids, as, e.g., the Gay-Berne potential (see [17] and references therein).

In spite of this great progress, for the reason of the simplicity, experimentalists often continue to interpret the results of their investigations in terms of the mean-field approximation [1]. The Landau-de Gennes theory as a phenomenological theory, where the order parameters are introduced without any reference to molecular properties, corresponds to the mean-field approximation. Prediction of this theory based on such macroscopic order parameters are in this sense universal. However, order parameters are often also constructed in relation to specific molecular models. They are called microscopic and by definition contain more information than just the symmetry of the phase. (When the molecules can be approximately taken as rigid, i.e., rigid rods, it is possible to find a connection between the macroscopic tensors of order parameter, defined through the susceptibility or the dielectric constant, to the microscopic quantities.) Therefore, different models are proposed to study the behaviour of orientational ordering [1-3].

Instead of analytical models with anisotropic shapes of molecules, different from ellipsoidal one, or numerical models with anisotropic interaction potentials, in our model the hard molecules have either a symmetrical ellipsoidal configu- 
ration or an asymmetrical ellipsoidal one and they are confined to the sites of a cubic lattice. The other sites are taken either empty $[4,18]$ or filled by spherical molecules [5]. These molecules have a continuous distribution of orientations in the space and there is no external (electric or magnetic) field. Also, this model in the case of very long symmetrical ellipsoidal molecules and in presence of an isotropic interaction among them, related to the orientational ordering, is equivalent to the lattice model of rod (cylindrical) molecules. In our approach, except the Landau-de Gennes expansion in Sec. 2.2.1, we can establish some relations among the interaction constants and relate the "shape" of ordering to the parameters of the model, particularly to the microscopic shape of the ellipsoidal molecules. Besides the orientational ordering in nematics studied and before [3], the new achievements in this work are the possibility of obtaining a biaxial ordered liquid state in the case of the system of ellipsoids, which are not axially symmetric; the orientational glassy structure and a simple explanation of orientational ordering in solid hydrogen (orth $-\mathrm{H}_{2}$ or para- $\mathrm{D}_{2}$ ) and, also, the presence of an ordered (uniaxial) phase of a disc-like (oblate) type when the temperature and the concentration are decreased in the system of nonmigrating ellipsoids.

\section{The lattice model of migrating molecules}

\subsection{The uniaxial phase}

\subsubsection{The lattice of ellipsoidal molecules}

Consider the system of axially symmetric ellipsoids dispersed on a cubic lattice, occupying all lattice points. The interactions are restricted to nearest neighbours. (In addition, in order to have later a possibility of a biaxial phase, these ellipsoids are considered not axially symmetric.) The components of the tensor, reflected this kind of molecule, in a proper frame of reference are given as diagonal elements of the matrix $Q$ :

$$
\left.\left.(Q)_{\alpha \beta}=Q_{\alpha \beta}(\alpha, \beta=x \text { (or } 1), y(\text { or } 2), z \text { (or } 3\right)\right), \quad \operatorname{Tr} Q=1,
$$

where $Q_{x x}=Q_{11}=Q_{1}, Q_{y y}=Q_{22}=Q_{2}$ and $Q_{z z}=Q_{33}=Q_{3}$. Or, for a traceless (symmetric second-rank) tensor we have

$$
q=Q-\frac{1}{3} I, \quad \operatorname{Tr} q=0,
$$

where $I$ is the unity tensor and $q_{i}=Q_{i}-\frac{1}{3}(i=1,2,3)$. (In the case of axially symmetric ellipsoids we have $Q_{1}=Q_{2}=Q$ or $q_{1}=q_{2}=q$.)

The Hamiltonian of the system, if we use the Einstein summation convention, is

$$
H=-\frac{1}{2} \sum_{i, j(\mathrm{n}-\mathrm{n})} J_{\alpha \beta \gamma \delta}^{i j} Q_{\alpha \beta}^{i} Q_{\gamma \delta}^{j}
$$

where $J_{\alpha \beta \gamma \delta}$ are the coupling constants of a four-rank tensorial interaction, while $i, j(\mathrm{n}-\mathrm{n})$ denotes summation over nearest-neighbour pairs of sites.

In the simple case of an isotropic interaction $J(J>0)$, treating this Hamiltonian in the molecular field approximation we find a trivial solution, corresponding to the isotropic state and another solution, describing a nematic phase. In this approximation, the averages or expectation values of $Q_{\alpha \beta}$ 's, the molecular 
parameters, referred to a coordinate system fixed in space (axes of the molecular field), are $\eta_{\alpha}=\left\langle Q_{\alpha \alpha}\right\rangle_{0} \neq 0$ for $\alpha=x, y, z$ or $1,2,3$, respectively, while all other components are zero. (The notation $\langle\ldots\rangle_{0}$, used above, means the average in the mean-field approach, based on the trial Hamiltonian $H_{0}$; further for the simplification of writing, the subscript 0 will be omitted.) For axially symmetric molecules we have also $\eta_{1}=\eta_{2}$ and $\eta_{3}=\eta=1-2 \eta_{1}$. Thus, $\eta=\eta_{3}$ can be used as an order parameter for the orientational ordering. This parameter has a discontinuous jump at the transition temperature $\tau_{Q} \approx 0.294(1-3 Q)^{2}$ (with $\tau=k_{\mathrm{B}} T / 3 J\left(k_{\mathrm{B}}\right.$ is the Boltzmann constant and $T-$ the absolute temperature)), from $\eta=1 / 3$ in the isotropic phase to some value $\eta \neq 1 / 3(>1 / 3)$ in the orientational phase. We can define also the order parameter as $s=3 \eta-1$ or, according to Maier and Saupe [19], as $S_{\mathrm{M}-\mathrm{S}}=(3 \eta-1) / 2(1-3 Q)$. The Maier-Saupe order parameter has a jump from $S_{\mathrm{M}-\mathrm{S}}=0$ to the value $S_{\mathrm{M}-\mathrm{S}} \approx 0.43$, which is in good agreement with many experiments (see, e.g., [1]), and in the completely orientational phase takes the value 1 (in this case $\eta=1-2 Q$ ).

We find the same result and for the tensor interaction $J_{\alpha \beta \gamma \delta}$, but in this case some geometrical restrictions are imposed [4]. Therefore, in the case of an interaction tensor of second rank $\left(-J_{\alpha \gamma}^{i j} Q_{\alpha \beta}^{i} Q_{\gamma \beta}^{j}\right)$, taking into account the symmetry properties of the energy of two interacting ellipsoids there are two nonvanishing constants $J_{1}$ and $J_{2}$. The interaction tensor in this case has the components: $J_{x x}=J_{y y}=J_{1}, J_{z z}=J_{2}$ and the other ones zero. In the molecular field approximation we obtain the same result as for the isotropic interaction $J$ with its value $J=2\left(2 J_{1}+J_{2}\right) / 6$. The model in this case leads to a possible arrangement of the long-range nematic order if $J_{1}>0, J_{2}<0$ and $J_{1}+J_{2}>0$. In the case when the interaction is a tensor of rank four (i.e. with $3^{4}=81$ components), taking into account the symmetry properties we find that there are very few independent interaction constants and concretely five nonvanishing independent coupling constants: $J_{x x x x}=J_{y y y y}=J_{1}, J_{z z z z}=J_{2}, J_{x x z z}=J_{y y z z}=J_{3}$, $J_{x x y y}=J_{4}, J_{x z x z}=J_{y z y z}=J_{5}$ and $J_{x y x y}=0.5\left(J_{1}-J_{4}\right)$. Studying the case when the lattice is completely full, we find the same self-consistency condition and an expression of the thermodynamic potential (in the unity of $3 J$ ), where a complementary term $-J^{\prime} / 6 J$ is present. These two new constants are connected to five independent interaction constants by the formulas: $6 J=4\left(2 J_{5}+J_{6}\right)$ and $J^{\prime}=\frac{2}{3}\left[\left(2 J_{1}+J_{2}\right)+2\left(2 J_{3}+J_{4}\right)-2\left(2 J_{5}+J_{6}\right)\right]$ for the direction [111]; $6 J=2\left(2 J_{1}+J_{2}-2 J_{3}-J_{4}\right)$ and $J^{\prime}=2\left(2 J_{3}+J_{4}\right)$ for the direction [001] with condition $2 J_{1}+J_{2}>2 J_{3}+J_{4}+2\left(2 J_{5}+J_{6}\right)$ or for an arbitrary direction with condition $2 J_{1}+J_{2}=2 J_{3}+J_{4}+2\left(2 J_{5}+J_{6}\right)$. By considering the nematic arrangement of the ellipsoids we obtain the following relations among the coupling constants: $J_{1}>J_{4}>J_{3}>J_{2}$ and $2 J_{3}<J_{1}+J_{2}\left(2 J_{1}+J_{2}>2 J_{3}+J_{4}\right)$.

\subsubsection{A partial equivalence of two models}

The model of ellipsoids only in the case of long symmetrical ellipsoidal molecules and in presence of an isotropic interaction among them, related to the orientational ordering, is equivalent to the lattice model of rod (cylindrical) molecules. In this case the molecular parameters, referred to a coordinate system fixed in space (axes of molecular field), are given by $R(\theta, \phi, \psi) Q R^{-1}(\theta, \phi, \psi)$ where 
$R$ is rotation matrix, determined by the Euler angles $(\theta, \phi, \psi)$ defining the molecular orientation in the mean field frame of reference. Explicitly, after calculations, we find

$$
\begin{aligned}
& Q_{x x}=Q+(1-3 Q) \cos ^{2} \theta \sin ^{2} \phi, \quad Q_{y y}=Q+(1-3 Q) \sin ^{2} \theta \sin ^{2} \phi \\
& Q_{z z}=Q+(1-3 Q) \cos ^{2} \phi, \quad Q_{x y}=(1-3 Q) \sin \theta \cos \theta \sin ^{2} \phi, \\
& Q_{x z}=(1-3 Q) \cos \theta \sin \phi \cos \phi, \quad Q_{y z}=(1-3 Q) \sin \theta \sin \phi \cos \phi .
\end{aligned}
$$

For a system of rods or cylindrical molecules, the components of the vector, representing this kind of molecule, in its proper frame of reference, are given by elements: $0,0,1$ of a column matrix $u_{0}$. In the axes of the molecular (mean) field we have $u=R u_{0}$ or

$$
u_{x}=\cos \theta \sin \phi, \quad u_{y}=\sin \theta \sin \chi, \quad u_{z}=\cos \phi .
$$

Substituting (5) in (4) we obtain

$$
\begin{aligned}
& Q_{x x}=Q+(1-3 Q) u_{x}^{2}, \quad Q_{y y}=Q+(1-3 Q) u_{y}^{2}, \quad Q_{z z}=Q+(1-3 Q) u_{z}^{2}, \\
& Q_{x y}=(1-3 Q) u_{x} u_{y}, \quad Q_{x z}=(1-3 Q) u_{x} u_{z}, \quad Q_{y z}=(1-3 Q) u_{y} u_{z} .
\end{aligned}
$$

In the particular case of an isotropic interaction $\left(J_{\alpha \beta \gamma \delta}=J \delta_{\alpha \gamma} \delta_{\beta \delta} \forall \alpha, \beta, \gamma, \delta\right)$, the Hamiltonian term can be written

$$
-J_{\alpha \beta \gamma \delta}^{i j} Q_{\alpha \beta}^{i} Q_{\gamma \delta}^{j}=-J Q_{\alpha \beta}^{i} Q_{\alpha \beta}^{j}=-J(2-3 Q) Q-J(1-3 Q)^{2}\left(u_{i} u_{j}\right)^{2} .
$$

The first constant term in (7) does not play a role in orientational ordering, while the second term is the Maier-Saupe interaction term.

\subsubsection{Lattice gas of ellipsoids}

We consider now a lattice gas model of ellipsoidal molecules to study the successive transitions according to the scheme: $\operatorname{gas}(G) \rightarrow$ isotropic liquid $(I) \rightarrow$ nematic liquid $(N)$ [4a]. For this model with migrating molecules and not fixed concentration, the Hamiltonian may be written in the following general form:

$$
H=-\frac{1}{2} \sum_{i, j(\mathrm{n}-\mathrm{n})} J_{\alpha \beta \gamma \delta}^{i j}\left(\sigma_{i} Q_{\alpha \beta}^{i}\right)\left(\sigma_{j} Q_{\gamma \delta}^{j}\right)-\mu \sum_{i} \sigma_{i}
$$

where $\sigma_{i}=1$ or 0 (the site $i$ is occupied or not) and $\mu$ is the chemical potential.

Studying this Hamiltonian (for the isotropic interaction) in the mean-field approximation we find two possibilities:

1) Diminishing the temperature, the system first undergoes a transition to the isotropic liquid state $(I)$ and then at lower temperature will make the transition to the nematic phase $(N)$. (For illustration in Fig. 1 there is represented the case when the value of molecular parameter is $Q=0.1$. There the condensation curve intersects the isotropic $\rightarrow$ nematic transition curve in a triple point. At temperatures below the triple point, only the transition curve gas $\rightarrow$ nematic does exist.)

2) With decreasing temperature the system goes directly from the gas phase to the nematic state, concretely for $Q \leq Q_{l}=0.056$ (the ellipsoid is very long); we have only one transition: gas $(G) \rightarrow$ uniaxial ordered liquid $(N)$. 


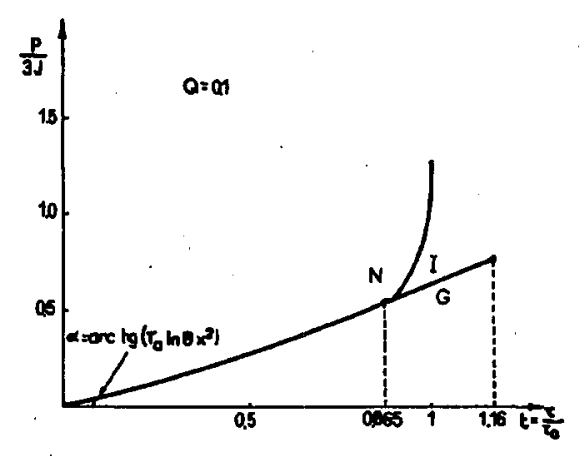

Fig. 1. The pressure-temperature phase diagram for molecular parameter $Q=0.1$ ( $G$ - the gas phase, $I-$ the isotropic state and $N$ - the nematic state).

In the case of the four-rank tensorial interaction we find that we must add a complementary term $\left(J^{\prime} / 6 J\right)$ (density) ${ }^{2}$ to the thermodynamic potential and another one $-2\left(J^{\prime} / 6 J\right)$ (density) to the chemical potential, but the main physics is the same. The new result here is that for $(1 / 6)\left(1+J^{\prime} / 6 J\right) /\left[(1 / 3.406)(1-3 Q)^{2}\right]>1$ or $J^{\prime} / 6 J>0.763$ there is no $Q_{l}$.

\subsection{The biaxial phase}

Considering the fact that the main lines of physical results in two cases analysed above are similar when we have an isotropic interaction or a four-rank tensorial interaction, for the reason of simplicity, to study the transition: uniaxial order $\rightarrow$ biaxial order we have taken the Hamiltonian

$$
H=-\frac{1}{2} \sum_{i, j(\mathrm{n}-\mathrm{n})} J Q_{\alpha \beta}^{i} Q_{\alpha \beta}^{j}
$$

with $J>0$ (such isotropic potential is not entirely realistic, particularly for the system of asymmetrical ellipsoidal molecules, but it does retain the essential physics of the problem).

\subsubsection{Phenomenological theory}

The transition between phases of different symmetry could be described in terms of order parameter, which is a symmetric traceless second-rank tensor. It is always possible to find a frame of reference, where it is diagonal. In this frame of reference its components are given as diagonal elements of the order matrix $S$ :

$$
\begin{aligned}
& S_{11}=\left\langle q_{11}\right\rangle=\frac{\eta}{\sqrt{6}}+\frac{\zeta}{\sqrt{2}}, \quad S_{22}=\left\langle q_{22}\right\rangle=\frac{\eta}{\sqrt{6}}-\frac{\zeta}{\sqrt{2}}, \\
& S_{33}=\left\langle q_{33}\right\rangle=-2 \frac{\eta}{\sqrt{6}} .
\end{aligned}
$$

The other non-diagonal components are zero. The state with $\eta \neq 0, \zeta \neq 0$ is a biaxial nematic state, which occurs in addition to the uniaxial phase $\eta \neq 0, \zeta=0$ and the isotropic phase $\eta=0, \zeta=0$. 
The free energy being a scalar, the expansion of this function in powers of the order parameter can only contain terms which are invariant combinations of the elements $S_{\alpha \beta}=\left\langle q_{\alpha \beta}\right\rangle$ of the order parameter tensor. In the language of group theory we have found these invariants, expressed by only two invariants

$$
\begin{aligned}
& I_{2}=\operatorname{Tr}\left(S^{2}\right)=\eta^{2}+\zeta^{2}, \quad I_{3}^{\prime}=\operatorname{Tr}\left(S^{3}\right)=(1 / 6)\left(3 \eta \zeta^{2}-\eta^{3}\right) \\
& \left(I_{3}=\eta^{3}-3 \eta \zeta^{2}\right), \quad I_{4}=\operatorname{Tr}\left(S^{4}\right)=(1 / 2) I_{2}^{2}, \quad I_{5}=\operatorname{Tr}\left(S^{5}\right)=(5 / 6) I_{2} I_{3}^{\prime}, \\
& I_{6}^{\prime}=I_{2}^{3}, \quad I_{6}^{\prime \prime}=\operatorname{Tr}\left(S^{6}\right)=(1 / 4) I_{2}^{3}+(1 / 2) I_{3}^{\prime 2} .
\end{aligned}
$$

In Landau-de Gennes theory of the nematic-isotropic phase transitions, Gramsbergen et al. [2] have constructed all absolute rotational invariants (ARI), which for any symmetric $3 \times 3$ matrix $S$, together with the traceless condition, are reduced to the invariants given by (11). The free energy expressed through these invariants is

$$
\begin{aligned}
F^{(6)} & =F(0)+(A / 2) I_{2}+(B / 3) I_{3}+(C / 4) I_{2}^{2}+D I_{2} I_{3}+(E / 6) I_{2}^{3} \\
& +(F / 6) I_{3}^{2}+\ldots
\end{aligned}
$$

The equilibrium states can be obtained from the minimum of the free energy with respect to $I_{2}$ and $I_{3}$ (or $\eta$ and $\zeta$ ). It is easy to see that the presence of the last term in (12) introduces the possibility of a biaxial state. Also Vigman et al. $[20,21]$ have shown that the width of the biaxial phase region is determined by the sixth-order term of the free energy expansion. Nevertheless, the biaxiality of the molecules forming an uniaxial thermotropic nematic can lead to renormalization of the Landau-de Gennes expansion coefficients, effectively diminishing or disappearing the biaxial ordering. The influence of molecular flexibility may be another factor leading to this kind of renormalization (see [21] and references therein). It is possible, also, to analyse the influence of the inclusion of the density variation in the Landau-de Gennes theory [22] related to this renormalization.

\subsubsection{Mean field theory}

In the absence of fields, a biaxial nematic phase may be expected only for molecules which are not axially symmetric (not even "effectively"). In this case, in the mean-field (m.f.) approximation, the trial Hamiltonian is

$$
H_{0}=-\sum_{i} E_{\alpha \beta}^{i} Q_{\alpha \beta}^{i}=-E_{\alpha} \sum_{i} Q_{\alpha \alpha}^{i}
$$

with $E_{x x}=E_{x}=E_{1}=E+\delta E=6 J\left\langle Q_{x x}\right\rangle=6 J \eta_{x}, E_{y y}=E_{y}=E_{2}=E-\delta E=$ $6 J\left\langle Q_{y y}\right\rangle=6 J \eta_{y}, E_{z z}=E_{z}=E_{3}=6 J-2 E=6 J\left\langle Q_{z z}\right\rangle=6 J \eta_{z}$.

In this approximation we have as before: $Q^{\text {m.f. }}=R(\theta, \phi, \psi) Q R^{-1}(\theta, \phi, \psi)$, where $Q_{1}=Q+\Delta Q, Q_{2}=Q-\Delta Q, Q_{3}=1-2 Q$ and $(\theta, \phi, \psi)$ are, as before, the Euler angles defining the molecular orientation in the mean field frame of reference. In m.f. approximation for the free energy we have 


$$
\frac{F^{\mathrm{m} . \mathrm{f}} \cdot / N}{3 J}=\frac{f}{3 J}=-\tau \ln Z_{0}+\left[\frac{s_{z}^{2}}{2}+\frac{s_{x y}^{2}}{2}+\left(1-s_{z}\right)^{2}\right], \tau=\frac{k_{\mathrm{B}} T}{3 J},
$$

where the partition function is

$$
Z_{0}=\int_{0}^{2 \pi} \mathrm{d} \psi \int_{0}^{2 \pi} \mathrm{d} \theta \int_{-1}^{1} \exp \left(\frac{2 I}{\tau}\right) \mathrm{d} X
$$

with

$$
\begin{aligned}
X= & \cos \phi, \quad I=Q+(1-3 Q)\left(1-\frac{X^{2}}{2}\right)-\frac{\Delta Q}{2}\left(1-X^{2}\right) \cos 2 \psi \\
& -\frac{\eta_{z}}{2}\left[\left(1-3 X^{2}\right)(1-3 Q)-3\left(1-X^{2}\right) \Delta Q \cos 2 \psi\right] \\
& +\frac{\delta E}{6 J}\left[(1-3 Q-\Delta Q \cos 2 \psi)\left(1-X^{2}\right) \cos 2 \theta\right. \\
& +2 \Delta Q(\cos 2 \theta \cos 2 \psi-2 X \sin 2 \theta \sin 2 \psi)]
\end{aligned}
$$

and the other parameters related to the ordering are

$$
s_{x y}=\eta_{x}-\eta_{y}, \quad s_{z}=1-\eta_{z} .
$$

Minimizing the free energy with respect to the components of the order parameter we find two self-consistency equations

$$
3 s_{z}-2=\tau \frac{\partial}{\partial s_{z}} \ln Z_{0}, \quad s_{x y}=\tau \frac{\partial}{\partial s_{x y}} \ln Z_{0},
$$

which can be solved numerically. From numerical analysis we have found three solutions: isotropic state, rotational symmetry state (or uniaxial state) and biaxial state. The transition from the isotropic phase to an uniaxial one is a first-order transition. For the biaxial solution the accuracy of triple integrals was not good and we have attempted an expansion of the exponential for low values of $\Delta Q$. For example, for $Q=0.15$ and $\Delta Q=0.01$ we have found for the biaxial order transition point: $\tau=0.076, s_{z}=1-\eta_{z}=0.42$ (or $\approx 0.34$ for the Maier-Saupe order parameter). The presence of a biaxial state and the decrease in the value of the order parameter could be useful for the explanation of ordering in the lipid bilayers. (It is commonly assumed that $S_{11}=S_{x x}=S_{22}=S_{y y}$. This implies either that the molecule has cylindrical symmetry about its $z$-axis or that it rotates about that axis in a time short compared with the time scale of the experiment. Both experimental and theoretical works suggest that for lipid chains in bilayers the $x$ and $y$-axes are not equivalent as far as motion is concerned [23].)

Also, in the case of a constant infinite-range isotropic interaction we have found two uniaxial phases, which are separated by a biaxial phase [4b]. Another system in which a biaxial phase could be expected is the mixture of rod-like and disc-like molecules $[24,25]$. In this case there is no need to go to low temperatures or high pressures, thus there is no risk of unwanted crystallization or smectic ordering as for our model described above. Also, a calculation based on the Potts-Ising model [26] predicts a biaxial phase in a mixture of prolate and oblate molecules. More theoretical and experimental work is necessary to understand better the behaviour of such binary mixtures or the ellipsoid-sphere mixtures. These systems 
seem to be important in the study of the dynamics of random interfaces [27] or the plastic state [5b]. We have used also the mixture of sphere-like ( $\mathrm{Cu}$ atoms) and ellipsoid-like ( $\mathrm{Zn}$ atoms) particles to interpret two transitions in the brass' diagram [28].

\section{The model of nonmigrating molecules}

\subsection{Quadrupolar or ellipsoidal glasses}

Consider the electrostatic quadrupole-quadrupole interaction, which is responsible for the orientational ordering in the solid hydrogen or in the mixture of ortho- and parahydrogen molecules [29]. Following [7,30,18], the electric quadrupole Hamiltonian can be written as

$$
H_{q q}=\frac{1}{2} \sum_{i, j} \sum_{m, n} \chi_{i j}^{m n} O_{i}^{m} O_{j}^{n},
$$

where $O_{i}^{m}$ are the operator equivalents of the spherical harmonics $Y_{2}^{m}$ in the manifold $|J|=1$, i.e., $O_{i}^{0}=\left(3 J_{z i}^{2}-2\right) / 3$ with $J_{z i}$ the $z$-axis component of the orbital angular momentum $\boldsymbol{J}$ on the site $i$, while $\chi_{i j}^{m n}$ are interaction constants.

In the internal-field approximation, the fluctuation terms are discarded to retain the truncated form

$$
H=\frac{1}{2} \sum_{i, j} \chi_{i j}^{00} O_{i}^{0} O_{j}^{0}
$$

where for nearest neighbours $\chi_{i j}^{00}=-19 \Gamma_{12} / 8\left(\Gamma_{12}=6 e^{2} Q_{\mathrm{u}}^{2} / 25 R_{12}^{5}, Q_{\mathrm{u}}\right.$ is the molecular quadrupole moment, $e-$ the electron charge and $R_{12}$ designates the intermolecular separation) $[7,30]$.

For the orbital angular momentum $|J|=1$, there are $2|J|+1=3$ values of the $z$-axis component, $\boldsymbol{J}_{z}(=-1,0,+1)$, which, in the coordinate system of their eigenvectors, can be represented by the matrix: $\operatorname{diag}(-1,0,+1)$, or its square by: $\operatorname{diag}(1,0,1)$. Thus, the modified operator $O^{\prime 0}=-O^{0} / 3=\left(2-3 J_{z}^{2}\right) / 9$ can be represented by the matrix: $\operatorname{diag}(-1 / 9,+2 / 9,-1 / 9)$, or, after an axis transformation, by

$$
\boldsymbol{O}^{\prime 0}=\operatorname{diag}(-1 / 9,-1 / 9,+2 / 9)=\operatorname{diag}(q, q,-2 q)=q(q=-1 / 9) .
$$

From Eqs. (19), (20), for an arbitrary coordinate system we refind (3) as follows:

$$
H=\frac{1}{2} \sum_{i, j} \sum_{\alpha \beta} I_{i j} q_{\alpha \beta}^{i} q_{\alpha \beta}^{j}
$$

where the interaction constant $I_{i j}$ can be expressed through $\chi_{i j}^{00}$. Therefore, related to the orientational space ordering, the system of quadrupoles is equivalent to the system of ellipsoidal (or cylindrical) molecules. Following the main lines of the way given in [30-33], for the low concentration $c_{0}$, below a critical temperature $T_{\mathrm{eg}} \approx 0.53 c_{0}^{1 / 2}$, we find a state, similar to a glassy structure (an orientational glass state, i.e., a "higher rank" glass state), in which all possible orientations of ellipsoids are equally likely, but the orientation at a given site is frozen [18]. These results seem to be useful for the description of the experimental facts related to the orientational ordering in such systems as solid hydrogen, by applying a model of ellipsoidal molecules. 


\subsection{The orientational ordering in solid hydrogen}

Let us have $0<c<6, c$ is the concrete number of nearest neighbours and $c_{0}=c / 6$ - the concentration. To study the variation of the transition temperature versus the concentration we shall suppose that there are no displacements of the ellipsoidal molecules from one site to another. For this model of nonmigrating molecules, assuming these objects as symmetrical ellipsoids, represented in its proper axes by tensor $q$ with $\operatorname{Tr} q=0$, the Hamiltonian is

$$
H=-\frac{1}{2} \sum_{i, j(c)} J \sigma_{i}^{0} \sigma_{j}^{0} q_{\alpha \beta}^{i} q_{\alpha \beta}^{j}
$$

where $\sigma_{i}^{0}$ has a given (fixed) value: 1 or 0 in the site $i$.

In the m.f. approximation following the same procedure as in Sec. 2.2.2, we find, for the variation of the phase transition temperature versus the concentration, the relation $T_{\mathrm{c}}=$ const $\cdot c$, which is linear.

In the "supermolecular" field approximation, the first-order approximation is properly the mean field approach. Assuming the commutation between $H$ and trial Hamiltonian $H_{0}$, the partition function can be written

$$
Z=\operatorname{Tr}\left[\exp (-\beta)\left(H-H_{0}\right) \exp \left(-\beta H_{0}\right)\right] \quad\left(\beta=\frac{1}{k_{\mathrm{B}} T}\right)
$$

and the free energy

$$
\begin{aligned}
F= & -\frac{1}{\beta} \ln Z \\
& =F_{0}+m_{1}\left(H-H_{0}\right)-\frac{\beta}{2} m_{2}\left(H-H_{0}\right)+\frac{\beta^{2}}{6} m_{3}\left(H-H_{0}\right)+\ldots
\end{aligned}
$$

where $m_{n}\left(H-H_{0}\right)$ is the $n$-th rank cumulant of $\left(H-H_{0}\right)$.

In fact, to find the free energy, we must calculate only the terms of $H$, the terms of $H_{0}$ can be found by a simple derivation. Therefore, i.e., we have

$$
\begin{aligned}
& m_{2}\left(H-H_{0}\right)=\left\langle H^{2}\right\rangle-\langle H\rangle^{2}-2\left(\left\langle H H_{0}\right\rangle-\langle H\rangle\left\langle H_{0}\right\rangle\right) . \\
& +\left\langle H_{0}^{2}\right\rangle-\left\langle H_{0}\right\rangle^{2}=\left\langle H^{2}\right\rangle-\langle H\rangle^{2}+2 \frac{\partial}{\partial \beta}\langle H\rangle+\frac{\partial^{2}}{\partial \beta^{2}} \ln Z_{0} .
\end{aligned}
$$

Analysing the terms of the free energy, we obtain

$$
\begin{aligned}
& m_{1}(H)=\langle H\rangle=-\frac{J}{2}\left\langle q_{\alpha \beta}\right\rangle^{2} N c \\
& m_{2}(H)=\left\langle H^{2}\right\rangle-\langle H\rangle^{2}=\frac{J^{2}}{4} \sum_{i j k l}\left(\left\langle q_{\alpha \beta}^{i} q_{\alpha \beta}^{j} q_{\gamma \delta}^{k} q_{\gamma \delta}^{l}\right\rangle-\left\langle q_{\alpha \beta}^{i} q_{\alpha \beta}^{j}\right\rangle\left\langle q_{\gamma \delta}^{k} q_{\gamma \delta}^{l}\right\rangle\right) .
\end{aligned}
$$

In the second case we have some common indexes for the terms of a nonzero expression in the bracket ("linked cluster theorem"). Then, to calculate $m_{2}(H)$ we consider separately two cases: (a) $j=k, i \neq l$ and (b) $j=k, i=l$.

In the case (a) there are 4 possibilities: $j=k, i \neq l ; j=l, i \neq k ; i=k, j \neq l$ and $i=l, j \neq k$, thus

$$
\begin{aligned}
& \left\langle H^{2}\right\rangle=J^{2} N c(c-1)\left\langle q_{\alpha \beta}\right\rangle\left\langle q_{\alpha \beta} q_{\gamma \delta}\right\rangle\left\langle q_{\gamma \delta}\right\rangle, \\
& \langle H\rangle^{2}=J^{2} N c(c-1)\left\langle q_{\alpha \beta}\right\rangle^{2}\left\langle q_{\gamma \delta}\right\rangle^{2} .
\end{aligned}
$$


In the case (b) there are 2 possibilities: $i=l, j=k$ and $i=k, j=l$, thus

$$
\begin{aligned}
& \left\langle H^{2}\right\rangle=\frac{1}{2} J^{2} N c\left\langle q_{\alpha \beta} q_{\gamma \delta}\right\rangle^{2}, \\
& \langle H\rangle^{2}=\frac{1}{2} J^{2} N c\left\langle q_{\alpha \beta}\right\rangle^{2}\left\langle q_{\gamma \delta}\right\rangle^{2} .
\end{aligned}
$$

To simplify the procedure, we exclude the condition $i \neq l$ in (a). The added term $(i=l)$ in (a) is subtracted in (b), which is very useful for a diagrammatic interpretation. From the relations

$$
\begin{aligned}
& m_{1}\left(q_{\alpha \beta}\right)=\left\langle q_{\alpha \beta}\right\rangle \quad\left(m_{1}\left(q_{\alpha \alpha}\right)=\left\langle q_{\alpha \alpha}\right\rangle=\eta_{\alpha}\right), \\
& m_{2}\left(q_{\alpha \beta} q_{\gamma \delta}\right)=\left\langle q_{\alpha \beta} q_{\gamma \delta}\right\rangle-\left\langle q_{\alpha \beta}\right\rangle\left\langle q_{\gamma \delta}\right\rangle, \text { etc } \ldots
\end{aligned}
$$

we obtain

$$
\begin{aligned}
& m_{1}(H)=\frac{1}{2} N c(-J) m_{1}^{2}\left(q_{\alpha \beta}\right)=\bullet-\bullet, \\
& m_{2}(H)=N c J^{2} m_{1}\left(q_{\alpha \beta}\right) m_{2}\left(q_{\alpha \beta} q_{\gamma \delta}\right) m_{1}\left(q_{\gamma \delta}\right)+\frac{1}{2} N c J^{2} m_{2}^{2}\left(q_{\alpha \beta} q_{\gamma \delta}\right) \\
& =\bigvee+\varnothing .
\end{aligned}
$$

To calculate $m_{n}(H)$ we follow these general rules:

1. consider all diagrams with $n$-linkings,

2. to each linking we join $-J$,

3. to each vertex with $k$-linkings, we join the cumulant $m_{k}\left(q_{\alpha \beta} q_{\gamma \delta} \ldots\right)$,

4. multiply by $(n ! / g)$, where $g$ is the rank of symmetry group of the graph,

5. make the total sum with respect to all ellipsoid indexes.

Following these rules and knowing that $\eta_{x}=\left\langle q_{x x}\right\rangle=\left\langle q_{y y}\right\rangle=\eta_{y}=-\frac{1}{2} \eta_{z}=$ $-\frac{1}{2}\left\langle q_{z z}\right\rangle,\left\langle q_{x y}\right\rangle=\left\langle q_{x z}\right\rangle=\left\langle q_{y z}\right\rangle=0,\left\langle q_{x y} q_{x z}\right\rangle=\left\langle q_{x y} q_{y z}\right\rangle=\ldots=0,\left\langle q_{\alpha \beta} q_{\gamma \delta}\right\rangle=0$ when $\alpha \neq \beta$ and $\gamma=x, y, z$, for the reduced free energy (per site), in the second-order approximation, we obtain the expression

$$
\begin{aligned}
& f_{0}=\left[f+(1 / \beta) \ln 8 \pi^{2}\right] /(3 / 2) J q^{2} \\
& =e_{z}-(1 / b) \ln I_{0}-0.5 c S^{2}+e_{z} S-0.75 c^{2} S^{2} \frac{\partial \eta_{z}}{\partial E_{z}^{0}}-(c / 2 b)\left[\left(\frac{\partial \eta_{x}}{\partial E_{x}^{0}}\right)^{2}\right. \\
& \left.+\left(\frac{\partial \eta_{y}}{\partial E_{x}^{0}}\right)^{2}+\left(\frac{\partial \eta_{z}}{\partial E_{z}^{0}}\right)^{2}\right]-0.75 e_{z}\left(e_{z}-2 c S\right) \frac{\partial \eta_{z}}{\partial E_{z}^{0}}-(4 / 9) c b\left(f_{1}^{2}+2 f_{2}^{2}\right)
\end{aligned}
$$

where

$$
\begin{aligned}
& b=(3 / 2) J \beta q^{2}, \quad e_{z}=-E_{z}^{0} / q=-E_{z} / q J, \quad S=-\eta_{z} / q=3 U-1, \\
& I_{0}=\int_{0}^{1} \exp \left(3 b e_{z} X^{2}\right) \mathrm{d} X, \quad U=I_{0}^{-1} \int_{0}^{1} X^{2} \exp \left(3 b e_{z} X^{2}\right) \mathrm{d} X \\
& V=I_{0}^{-1} \int_{0}^{1} X^{4} \exp \left(3 b e_{z} X^{2}\right) \mathrm{d} X
\end{aligned}
$$

and 


$$
\begin{aligned}
& \frac{\partial \eta_{z}}{\partial E_{z}^{0}}=6 b\left(V-U_{2}\right) \quad\left(E_{z}^{0}=E_{z} / J\right), \\
& \frac{\partial \eta_{x}}{\partial E_{x}^{0}}=0.75 b\left(1-2 U+3 V-2 U^{2}\right) \quad\left(E_{x}^{0}=E_{x} / J\right), \\
& \frac{\partial \eta_{y}}{\partial E_{x}^{0}}=0.75\left(-1+2 U+V-2 U^{2}\right), \\
& f_{1}=1-S+0.25 S^{2}+(1.5 / b) \frac{\partial \eta_{x}}{\partial E_{y}^{0}}=(9 / 8)(1-2 U+V) \quad\left(E_{y}^{0}=E_{y} / J\right), \\
& f_{2}=1+0.5 S-0.5 S^{2}-(3 / 4 b) \frac{\partial \eta_{z}}{\partial E_{z}^{0}}=(9 / 2)(U-V) .
\end{aligned}
$$

In the disordered (isotropic) phase, where $S=0\left(e_{z}=0\right)$, we have $f_{0}(S=0)=-0.8 c b$. From numerical determination of the absolute minimum of the free energy differences: $\Delta f_{0}(S)=f_{0}(S)-f_{0}(0)=f_{0}(S)+0.8 c b$, we find "the coordinates" of this minimum, i.e., the critical temperatures of the different phase transitions, for a given (fixed) value of $c$. It can be seen from Fig. 2a,b and Fig. 3a,b that the transitions: isotropic state $\rightarrow$ uniaxial state are of the first order. In Fig. 4 we have represented the variation of the reduced critical temperature $\tau_{\mathrm{c}}$ versus the concentration $c_{0}$, obtained by the method outlined above for the model of symmetrical ellipsoids. The most important feature of the studied system is the existence of a special critical point (a critical concentration $\approx 0.445$ ) clearly shown in Fig. 4. When the temperature and the concentration are decreased, the ordered (uniaxial) phase is a disc-like (oblate) phase $\left(\eta_{z}<0\right)$. The part 102 of the phase diagram in this figure reproduces conveniently the experimental results for the orientational ordering in solid hydrogen (ortho- $\mathrm{H}_{2}$ or para- $\mathrm{D}_{2}$ ) [7], while the part $O 3$ is not observed. Also, no percolation evidence is found in this case. If we consider free migration and also, the empty sites filled by spherical molecules as in [5], in the m.f. approximation and the approach of first (nearest) neighbours
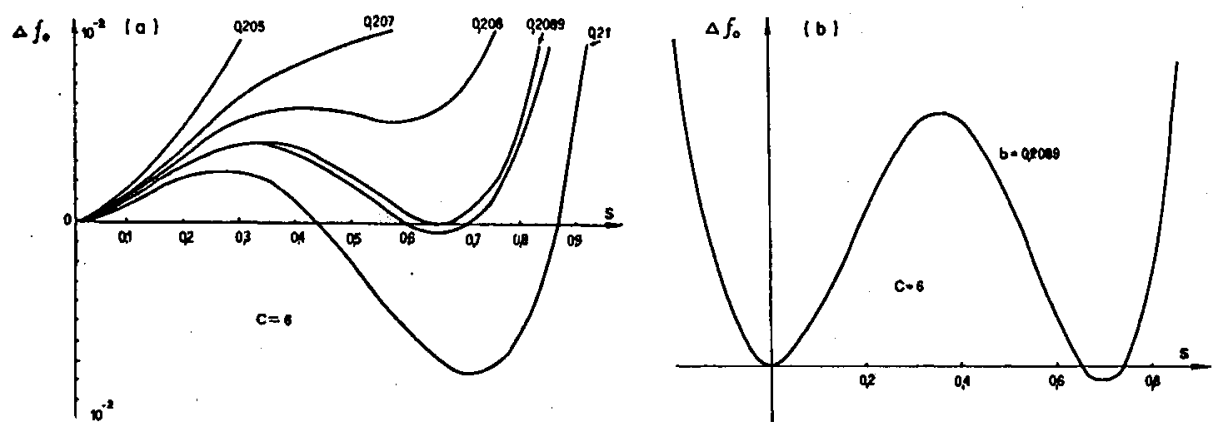

Fig. 2. The variation of the free energy difference $\Delta f_{0}$ versus the order parameter $S$ in the case $c=6$, (a) for different reduced temperatures, (b) near the critical temperature. 

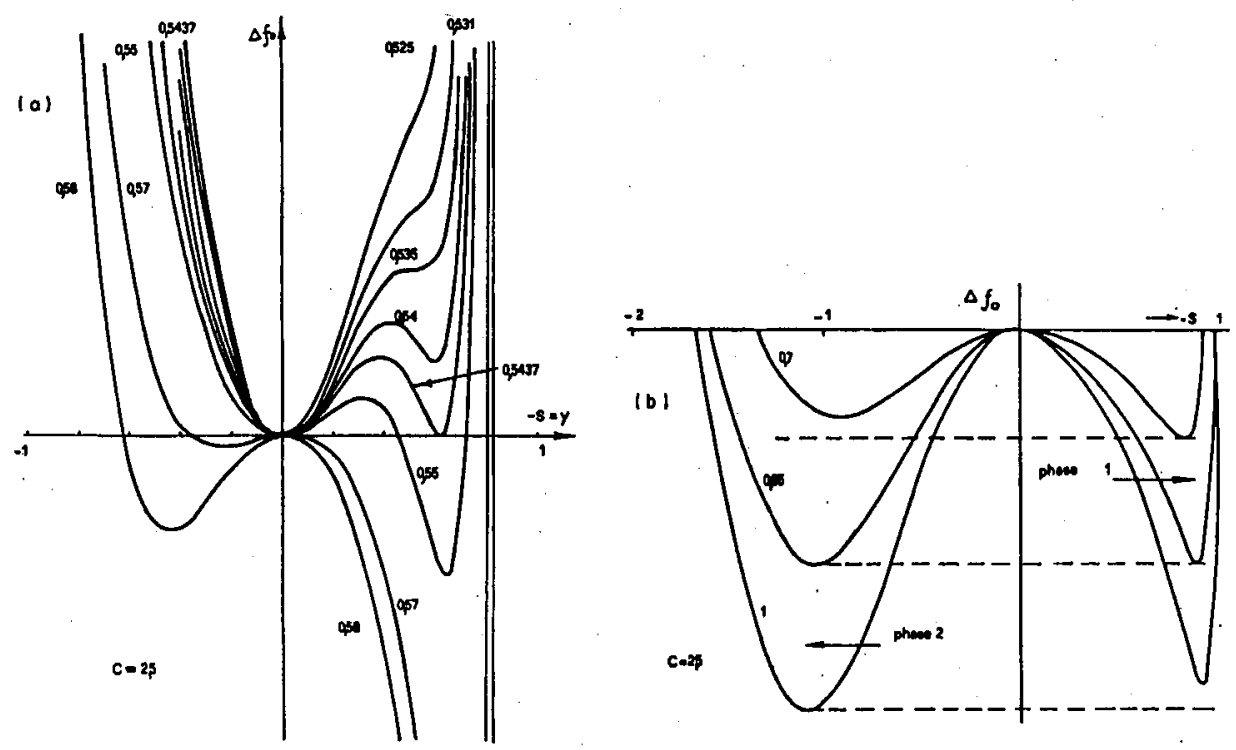

Fig. 3. (a) (b) The same variations as in Fig. 2a,b for the case $c=2.5$.

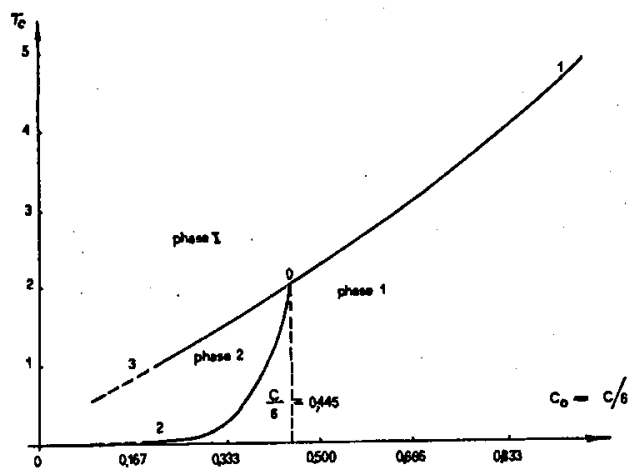

Fig. 4. The variation of the reduced critical temperature $\tau_{c}$ versus the concentration $c_{0}$.

interactions, we can find a kind of "percolation concentration" for the orientational ordering (dependent on the positional ordering), but the transition oblate phase $\rightarrow$ prolate phase will be not more possible.

\section{Comclusions}

This paper presents a simplified description of the orientational ordering of anisotropic particles of ellipsoidal (uniaxial or biaxial) symmetry, dispersed in a cubic lattice. The nearest-neighbour tensorial interactions, which correspond to these particles or bodies of ellipsoidal kind, are the simplest purely orientational ones, fully separated from the translational degrees of freedom. This simplification is necessary to avoid artifacts related to the space arrangements of the particles. 
The main aim of this paper was to study, within a standard analysis, the different orientationally ordered states and respectively different phase transitions in this system of asymmetrical (ellipsoidal) molecules (axially symmetric or not). The results, obtained by using a molecular or supermolecular field approach, are in good agreement with some experimental facts related to nematics, solid hydrogen, etc. Another result is the theoretical existence of a biaxial nematic mesophase, but up to now a biaxial nematic phase has not been observed for thermotropic liquid crystals, it has been discovered in soap-like solutions. Gas-liquid-nematic phase diagram and the orientational glass are also considered.

A new application of this molecular model could be possible in studying the biological membranes. Now we are trying to use this system of molecules, dispersed in a quasi-cubic (a bilayer) lattice, to explain the ordering and its variation in lipid bilayers.

\section{Acknowledgments}

The author would like to thank Prof. Yu Lu in Condensed Matter Physics Group, in ICTP (Trieste, Italy) for his permanent support.

\section{References}

[1] (a) P.-G. de Gennes, Scaling Concepts in Polymer Physics, Cornell University Press, Ithaca 1979; (b) P.-G. de Gennes, J. Prost, The Physics of Liquid Crystals, Clarendon Press, Oxford 1993.

[2] F. Gramsbergen, L. Longa, W.H. de Jeu, Phys. Rep. 135, 195 (1986).

[3] S. Chandrasekhar, Contemp. Phys. 29, 527 (1988).

[4] N. Boccara, R. Mejdani, L. De Seze, (a) Mol. Cryst. Liq. Cryst. 35, 91 (1976); (b) J. Phys. (Paris) 38, 149 (1977).

[5] (a) R. Mejdani, Phys. Status Solidi B 168, 113 (1991); (b) R. Mejdani, A. Gashi, Phys. Status Solidi B 186, 403 (1994).

[6] N.H. Tinh, C. Destrade, H. Gasparoux, Phys. Lett. A 72, 251 (1979).

[7] N.S. Sullivan, H. Vinegar, R.V. Pound, Phys. Rev. B 12, 2596 (1975).

[8] A. Wurflinger, Int. Rev. Phys. Chem. 12, 89 (1993).

[9] M. Yvinec, R.M. Pick, J. Phys. (Paris) 41, 1045 (1980); ibid. 44, 169 (1983).

[10] For reviews of this subject see D. Frenkel, Mol. Phys. 60, 1 (1987); M.P. Allen, Philos. Trans. R. Soc. Lond. A 344, 323 (1993).

[11] M.P. Allen, G.T. Evans, D. Frenkel, B.M. Mulder, Adv. Chem. Phys. 86, 1 (1993).

[12] M.P. Allen, M.R. Wilson, J. Comput. Aided Mol. Des. 3, 335 (1989); M.P. Allen, in: Proc. Int. School of Physics "Enrico Fermi", Course CXXIX, Varenna 1994, Eds. M. Baus, L. Rull, J.-P. Ryckaert, Kluwer, Dordrecht 1995.

[13] A.C. Branka, K.W. Wojciechowski, Mol. Phys. 72, 941 (1991); ibid. 78, 1513 (1993).

[14] Ordering in Supramolecular Fluids, Eds. D. Frenkel, H.N.W. Lekkerkerker, Th. Odijk, Periodical Collection, C1991, Workshop, Amsterdam 1990; Physica A 176, 1 (1991).

[15] M. Dijkstra, D. Frenkel, H.N.W. Lekkerkerker, Physica A 193, 374 (1993); M. Dijkstra, D. Frenkel, Phys. Rev. E 50, 349 (1994). 
[16] M.P. Allen, D. Frenkel, Phys. Rev. A 37, 1813 (1988); M.P. Allen, D. Frenkel, Phys. Rev. $A$ 42, 3641 (1990).

[17] D.J. Cleaver, C.M. Care, M.P. Allen, M.P. Neal, Phys. Rev. E 54, 559 (1996).

[18] R. Mejdani, Balkan Phys. Lett. 1, 15 (1993).

[19] W. Maier, A. Saupe, Z. Nat.forsch. A 13, 564 (1958); 14, 882 (1959); 15, 287 (1960); 16, 816 (1961).

[20] P.R. Vigman, A.I. Larkin, V.M. Filov, Sov. Phys.-JETP 68, 944 (1975).

[21] M.A. Anisimov, Critical Phenomena in Liquids and Liquid Crystals, Gordon and Breach, Singapore 1991, p. 313.

[22] P.K. Mukherje, T.R. Bose, D. Ghose, M. Saha, Phys. Rev. E 51, 4570 (1995).

[23] B.L. Silver, The Physical Chemistry of Membranes, Allen/Unwin and Solomon Press, New York 1985, Ch. 5, Ch. 8.

[24] L.J. Yu, A. Saupe, Phys. Rev. Lett. 45, 1000 (1980).

[25] Z.Y. Chen, J.M. Deutch, Chem. Phys. 80, 2151 (1984).

[26] R.G. Caflisch, Z.Y. Chen, A.N. Berker, J.M. Deutch, Phys. Rev. A 30, 2562 (1984).

[27] R. Hashim, G.R. Luckhurst, S. Romano, Mol. Phys. 53, 1535 (1984).

[28] R. Mejdani, Bull. Tec. Sci. 1, 15 (1980).

[29] T. Nakamura, O. Nagai, Prog. Theor. Phys. 24, 432 (1960).

[30] N.S. Sullivan, J. Phys. (Paris) 37, 981 (1976).

[31] D.J. Sherrington, J. Phys. C, Solid State Phys. 8, L208 (1975).

[32] B. Southern, J. Phys. C, Solid State Phys. 8, L213 (1975).

[33] D. Sherrington, S. Kirkpatrick, Phys. Rev. Lett. 35, 1792 (1975). 